\title{
Model-Based Determination of Effective Blood Concentrations of Cyclosporine for Neutrophil Response in the Treatment of Severe Aplastic Anemia in Children
}

\author{
Michaël Philippe, ${ }^{1,2,7}$ Emilie Hénin, ${ }^{2,3}$ Yves Bertrand, ${ }^{1}$ Dominique Plantaz, ${ }^{4}$ \\ Sylvain Goutelle, ${ }^{2,5,6}$ and Nathalie Bleyzac ${ }^{1,2}$
}

Received 19 January 2015; accepted 24 April 2015; published online 15 May 2015

\begin{abstract}
Optimal immunosuppressive therapy in acquired severe aplastic anemia (SAA) remains to be refined, especially cyclosporine (CsA) use. Current recommendations state that CsA trough blood concentrations (TBC) should be maintained between 200 and $400 \mathrm{ng} / \mathrm{mL}$ despite the lack of supporting data. This study aimed at quantifying relationships between CsA exposure and neutrophil response and determining an effective range for CsA TBC. Twenty-three SAA patients treated with CsA were retrospectively analyzed. Nonlinear mixed effect modeling approaches were used to develop a pharmacokinetic-pharmacodynamic model. The pharmacokinetic model described the relationships between CsA doses and TBC. The pharmacodynamic model allowed to estimate boundaries for optimal CsA effects, neutrophils being used as biomarker of response. A time-to-event model linked effective concentration to time-to-therapeutic success. CsA TBC were adequately described by a twocompartment model with first-order absorption, a lag time, and a linear elimination. The efficient range of CsA TBC was estimated between 87 and $120 \mathrm{ng} / \mathrm{mL}$. Model-based simulations and external validation in three additional patients confirmed these results. This original modeling approach was successful in describing the relationship between CsA TBC and neutrophil response in SAA patients. Although further evaluation of the model is necessary, this work suggests that an optimal CsA TBC target of $100 \mathrm{ng} / \mathrm{mL}$ would be associated with a better neutrophil response in children with SAA.
\end{abstract}

KEY WORDS: aplastic anemia; cyclosporine; modeling; pharmacokinetics; regulatory T lymphocytes.

${ }^{1}$ Institut d'Hématologie et d'Oncologie Pédiatrique, 1 place Joseph Renaut, 69008, Lyon, France.

${ }^{2}$ Laboratoire de Biométrie et Biologie Evolutive, UMR CNRS 5558, Université Lyon 1, 43 bd du 11 novembre 1918, 69622, Villeurbanne, France.

${ }^{3}$ Service de Pharmacologie et Essais Thérapeutiques, Faculté de Médecine Laennec, Hospices Civils de Lyon, Rue Guillaume Paradin, 69376, Lyon Cedex 08, France.

${ }^{4}$ Unité d'onco-hématologie pédiatrique, Hôpital La tronche, Université de Grenoble, Avenue du Maquis du Grésivaudan, 38700, Grenoble, France.

${ }^{5}$ Faculté de Pharmacie de Lyon, ISPB, Université Lyon 1, 8 avenue Rockefeller, 69373, Lyon, France.

${ }^{6}$ Service Pharmaceutique, Groupement Hospitalier de Gériatrie, Hospices Civils de Lyon, 136 Rue Commandant Charcot, 69005, Lyon, France.

${ }^{7}$ To whom correspondence should be addressed. (e-mail: michael. philippe@chu-lyon.fr)

ABBREVIATIONS: ANC, Absolute neutrophil count; ATG, Antithymocyte globulin; BMT, Bone marrow transplantation; BSA, Body surface area; BW, Body weight; CsA, Cyclosporine; hATG, Horse antithymocyte globulin; Hb, Hemoglobin; IIV, Interindividual variability; IST, Immunosuppressive therapy; OFV, Objective function value; PD, Pharmacodynamic; PK, Pharmacokinetic; PK-PD, Pharmacokinetic-pharmacodynamic; SAA, Severe aplastic anemia; SAEM, Stochastic approximation expectation maximization; TBC, Trough blood concentrations; Treg, Regulatory T cells; TTR, Time-to-response.

\section{INTRODUCTION}

Severe aplastic anemia (SAA) is a rare hematologic disease resulting in a quantitative bone marrow insufficiency. Currently, aplastic anemia affects one to two persons per million population per year $(1,2)$, with two incidence peaks, the first one between 10 and 25 years old and the second one over 60 years old (3). Without treatment, this disease is fatal, mainly from infectious diseases due to immunodepression. The first-line treatment is bone marrow transplantation (BMT) from a matched sibling donor. If no genoidentical donor is available, the alternative is an immunosuppressive therapy (IST) including antithymocyte globulin (ATG) and cyclosporine (CsA) (4-6). If no response is obtained, a phenoidentical allograft from voluntary donors is considered.

Since 2006, therapeutic recommendations have been as follows (7,8): horse ATG (hATG) $160 \mathrm{mg} / \mathrm{kg}$ then CsA $5 \mathrm{mg} / \mathrm{kg} / 12 \mathrm{~h}$ for at least 6 months, with dose adjusted to maintain trough blood concentrations (TBC) between 200 and $400 \mathrm{ng} / \mathrm{mL}$. This standard IST allows a hematological response at 6 months in about $60 \%$ of patients $(9,10)$.

However, uncertainties remain about the links between SAA physiopathology and the IST mechanism of action. The response to IST suggests an autoimmune mechanism (11), with the involvement of deficient regulatory $\mathrm{T}$ cells (Treg). Recently, it has been shown that Treg counts are reduced at diagnosis (12-15). Furthermore, Treg have been suggested as 
a prognostic factor for response to IST (16). CsA appears to have a dual effect on Treg in vivo, depending on the concentration. At low concentration, CsA stimulates the Treg proliferation, while it inhibits their development at higher concentration (17). This dual effect is not considered in the current recommendations for SAA treatment. In our experience, decreasing CsA exposure has been associated with a positive outcome in children who experienced an initial failure of IST (18). This suggests a potentially positive impact of decreasing CsA TBC in SAA treatment. However, the effective range for CsA TBC has not been determined yet.

The objective of our study was to develop mathematical models to characterize the relationships between exposure to CsA and neutrophil response, and to determine the effective range for CsA TBC in children with SAA.

\section{MATERIALS AND METHODS}

\section{Patient Recruitment}

This was a retrospective analysis of patients with SAA who were treated in our center from 1998 to 2013. SAA was defined by two of the following criteria: absolute neutrophil count $(\mathrm{ANC})<0.5 \times 10^{9} / \mathrm{L}$, platelets $<20 \times 10^{9} / \mathrm{L}$, or hemoglobin $<80 \mathrm{~g} / \mathrm{L}$ with reticulocytes $<20 \times 10^{9} / \mathrm{L}$ (19). Patients were included if the full history of the following data was available: doses and measured CsA TBC, comedications, blood cell count (red blood cells, hemoglobin, reticulocytes, platelets, neutrophils, and lymphocytes), transfusions, and anthropometric data. Minimum treatment duration was fixed to 3 months.

Three patients who experienced failure after a first treatment course received a second course of IST because of the unavailability of phenoidentical allograft from voluntary donors. In such cases, the two sequences were considered as two independent individuals. The ethical committee's opinion was not needed for this observational study, in accordance with French legislation (20).

\section{Immunosuppressive Regimens}

Patients received $160 \mathrm{mg} / \mathrm{kg}$ hATG or $18.75 \mathrm{mg} / \mathrm{kg}$ rabbit ATG. Initial CsA dose regimen was $5 \mathrm{mg} / \mathrm{kg} / 12 \mathrm{~h}$ per os. CsA TBC were monitored once a week or less in outpatients. The initial CsA target of TBC was $150 \mathrm{ng} / \mathrm{mL}$. CsA TBC were the same during the entire course of each patient. No granulocyte colony-stimulating factor has been used.

\section{Population Pharmacokinetic/Pharmacodynamic Model}

\section{Population Approach and Software}

Data were analyzed using nonlinear mixed effect modeling. Model building and parameter estimation were performed using the stochastic approximation expectation maximization (SAEM) algorithm $(21,22)$ implemented in the Monolix software (version 4.2.0; Lixoft) (23).

The interindividual variability (IIV) in the model parameters $(P$, e.g., CsA clearance or volume of distribution) was described using an exponential model, which implies an assumed log-normal distribution of the parameters between individuals in the population:

$P_{i}=P_{\mathrm{pop}} \cdot e^{\eta p_{i}}$

where $P_{i}$ is the parameter $P$ for the $i$ th individual, $P_{\text {pop }}$ is the typical (average) parameter value in the population, and $\eta_{\mathrm{p} i}$ is a random variable quantifying the variation from the typical (average) individual. The $\eta_{\mathrm{p} i}$ are assumed to be normally distributed, with a mean of zero and a variance of $\omega_{\mathrm{p}}^{2}$.

\section{Population Pharmacokinetic Model}

To describe the relationships between CsA doses and TBC, one-, two-, and three-disposition compartment models, with various elimination models (linear or nonlinear), were tested. Since no pharmacokinetic (PK) information in the absorption phase was available, parameters related to the absorption (bioavailability, lag time, and first-order absorption rate constant) were fixed, according to the literature (24).

The observed concentration value $\operatorname{Cobs}_{i j}$ in the $i$ th individual on the $j$ th occasion was linked to the corresponding

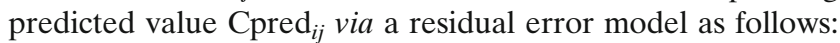

$\operatorname{Cobs}_{i j}=\operatorname{Cpred}_{i j}+w_{i j} \cdot \varepsilon_{i j}$

where $\varepsilon_{i j}$ is a random variable with a standard normal distribution, and $w_{i j}$ is the standard deviation of the residuals $\left(\mathrm{Cobs}_{i j}-\mathrm{Cpred}_{i j}\right)$. Different residual models were tested: additive or homoscedastic model $\left(w_{i j}=a\right)$; proportional or heteroscedastic model $\left(w_{i j}=b \cdot \operatorname{Cpred}_{i j}\right)$; or a combined model $\left(w_{i j}=\sqrt{a^{2}+b^{2} \cdot \text { Cpred }_{i j}^{2}}\right)$, where $a$ and $b$ are constants.

In order to explain the IIV, continuous $\left(\mathrm{COV}_{\text {cont }}\right)$ and categorical covariates $\left(\mathrm{COV}_{\text {cat }}\right.$ coded as 0 or 1$)$ were tested for potential effects on model parameters. The general equation of the relationship between parameter and those covariates was as follows:

$P_{i}=\left(P_{\mathrm{pop}}+\beta_{\mathrm{cat}} \cdot \mathrm{COV}_{\mathrm{cat}}+\beta_{\mathrm{cont}} \cdot\left(\mathrm{COV}_{\mathrm{cont}}-\operatorname{median}_{\mathrm{COV}} \mathrm{cont}\right)\right) \cdot e^{\eta_{i}}$

where $\beta_{\text {cat }}$ and $\beta_{\text {cont }}$ quantify the effect of covariate on the parameter $P$ distribution: the typical individual has $\mathrm{COV}_{\text {cat }}=0$ and $\mathrm{COV}_{\text {cont }}=$ median $_{\mathrm{COV} \text { cont }} . \beta_{\text {cat }}$ is the average increase in the parameter $P$ when $\mathrm{COV}_{\text {cat }}=1$ as compared to $\mathrm{COV}_{\text {cat }}=0$; $\beta_{\text {cont }}$ is the average increase in the parameter $P$ per unit of increase in $\mathrm{COV}_{\text {cont }}$.

The following covariates were tested on each parameter, following a stepwise forward-backward selection procedure: age, sex, body weight (BW), height, body surface area (BSA), creatinine clearance, and cotreatment with a potent cytochrome 3A4 enzyme inhibitor (azole antifungals, macrolide antibiotics, calcium channel blockers).

In the forward step, the covariates were tested in a univariate manner on each parameter and retained if they significantly improved the model fit (likelihood ratio test (LRT), $p<0.05)$. In the backward step, all significant covariates identified in the first step were included in the model 
and deleted sequentially. The covariates associated with a significant increase in the objective function (LRT, $p<0.01$ ) were kept in the final model.

In addition, BW was tested for potential effects on model parameters, those parameters being allometrically scaled to weight as follows:

$P_{i}=P_{\mathrm{pop}} \times\left(\mathrm{BW}_{i} / \mathrm{BW}_{\mathrm{pop}}\right)^{a} \cdot e^{\eta_{i}}$

where $\mathrm{BW}_{\text {pop }}$ is the average weight in this population, $\mathrm{BW}_{i}$ is the weight of the $i$ th individual, and $a$ is the allometric coefficient, being fixed to 0.75 for clearance parameters and 1 for volume parameters.

\section{Pharmacodynamic and Time-to-Event Model}

The response to treatment was defined as an ANC above $0.50 \times 10^{9} / \mathrm{L}$ observed on two consecutive occasions, and the time-to-response (TTR) was defined as the delay between CsA treatment initiation and the second occasion confirming ANC above $0.50 \times 10^{9} / \mathrm{L}$. ANC was monitored twice weekly until response. Response to drug therapy was monitored up to 6 months in all patients, as a phenoidentical allograft is usually considered when no favorable response is observed within this time frame. The response to treatment on other blood cells was reported separately and was not considered in the model building:

- Red blood cell response if hemoglobin count $>80 \mathrm{~g} / \mathrm{L}$ and transfusion independence

- Platelet response if platelet count $>20 \mathrm{G} / \mathrm{L}$ and transfusion independence

The main assumptions for model development were:

- There exists a delay between kinetics of CsA blood concentrations and kinetics of CsA effects.

- There is an effective range of CsA TBC: the hazard of response is lower when the TBC is outside a range, to be determined; the hazard of response is maximal for an optimal CsA TBC value, being the centered value of the range.

These assumptions were translated into equations using the interface model (25) and time-to-event approaches. The interface model describes the profile of an assumed effective concentration $\left(C_{\mathrm{e}}\right)$ in an effect compartment, as a nonlinear function of CsA TBC. The function was adapted to estimate two bounds of CsA TBC defining an optimal range as follows:

$\frac{\mathrm{d} C_{\mathrm{e}}}{\mathrm{d} t}=-\alpha \cdot C_{\mathrm{e}}+\sqrt{[(\text { Ctrough }-\gamma 1) \cdot(\gamma 2-\mathrm{Ctrough}) \cdot \mathrm{H} 1 \cdot \mathrm{H} 2]}$

where Ctrough is the CsA TBC predicted by the PK model, $\gamma 1$ and $\gamma 2$ are the lower and upper bounds of CsA TBC to be estimated by the algorithm, $\mathrm{H} 1$ and $\mathrm{H} 2$ are categorical variables whose values depend on Ctrough: $\mathrm{H} 1$ is equal to 1 if Ctrough is greater than $\gamma 1$ and $\mathrm{H} 2$ is equal to 1 if Ctrough is lower than $\gamma 2$, and $\mathrm{H} 1$ and $\mathrm{H} 2$ are equal to 0 otherwise. Ctrough, $\gamma 1$, and $\gamma 2$ are the input variables, and $\alpha$ is the output parameter (the elimination rate constant of effective concentration, to be estimated). As a result, $C_{\mathrm{e}}$ may eventually increase only if Ctrough value is between $\gamma 1$ and $\gamma 2$; otherwise, it may decrease. Figure 1 represents the pharmacokinetic-pharmacodynamic (PK-PD) model structure.

A schematic representation of a typical time profile of CsA TBC (Ctrough) and the corresponding time profile for the effective concentrations $\left(C_{\mathrm{e}}\right)$ are given in Fig. 2. The effective concentration increases when Ctrough is included in the interval $\gamma 1-\gamma 2$, and is eliminated with a rate constant $\alpha$.

The TTR was modeled using time-to-event approaches (26). The instantaneous hazard $\lambda(t)$ is defined as the hazard of response to treatment at time $t$ given that the patient had not responded at time $t-\mathrm{d} t(\mathrm{~d} t$ approaching 0$)$. In our model, the instantaneous hazard was assumed to increase with the effective concentration $C_{\mathrm{e}}$. The equation linking the instantaneous hazard with the effective concentration $C_{\mathrm{e}}$ was as follows:

$\lambda(t)=\lambda_{0} \cdot\left(1+\right.$ slope $\left.\cdot C_{\mathrm{e}}(t)\right)$

where $\lambda_{0}$ is the instantaneous hazard of response without treatment $\left(C_{\mathrm{e}}=0\right)$ and slope, constrained to be positive, is the rate of increase of the instantaneous hazard for one unit increase of $C_{\mathrm{e}}$.

The probability density function for the distribution of events $F(t)$, i.e., response to CsA treatment can be expressed as a function of the integrated hazard through time, taking therefore into account the whole profile of effective concentrations until time $t$ :

$F(t)=1-\exp \left(-\int_{0}^{t} \lambda(u) \cdot \mathrm{d} u\right)$

In case of a patient not receiving effective concentrations of CsA ( $C_{\mathrm{e}}=0$ at any time), the expression of the probability density function $F(t)$ can be simplified to:

$F(t)=1-\exp \left(-\int_{0}^{t} \lambda(u) \cdot \mathrm{d} u\right)=1-\exp \left(-\int_{0}^{t} \lambda_{0}\right)=1-\exp \left(-\lambda_{0} \cdot t\right)$

\section{Model Evaluation}

First, the quality of parameter estimation was assessed using the parameter uncertainty (standard errors obtained from a stochastic approximation of the Fisher information matrix) and the SAEM algorithm convergence. Secondly, we analyzed the goodness-of-fit criteria: comparison of population and individual predictions to observations, distribution of residuals and individual parameters, and minimization of the objective function value $(\mathrm{OFV}=-2 \mathrm{log}$ likelihood). The addition of a parameter, e.g., the effect of one covariate on a PK parameter, into the model significantly improved the fit if an OFV drop greater than 3.84 with an $\alpha$ risk at 5\% was observed for a candidate model, compared to a reference model. Finally, simulation-based diagnostics were performed with visual predictive checks where replicates of the original data are simulated given the model structure and parameter distributions and then compared to the observed data (27). 


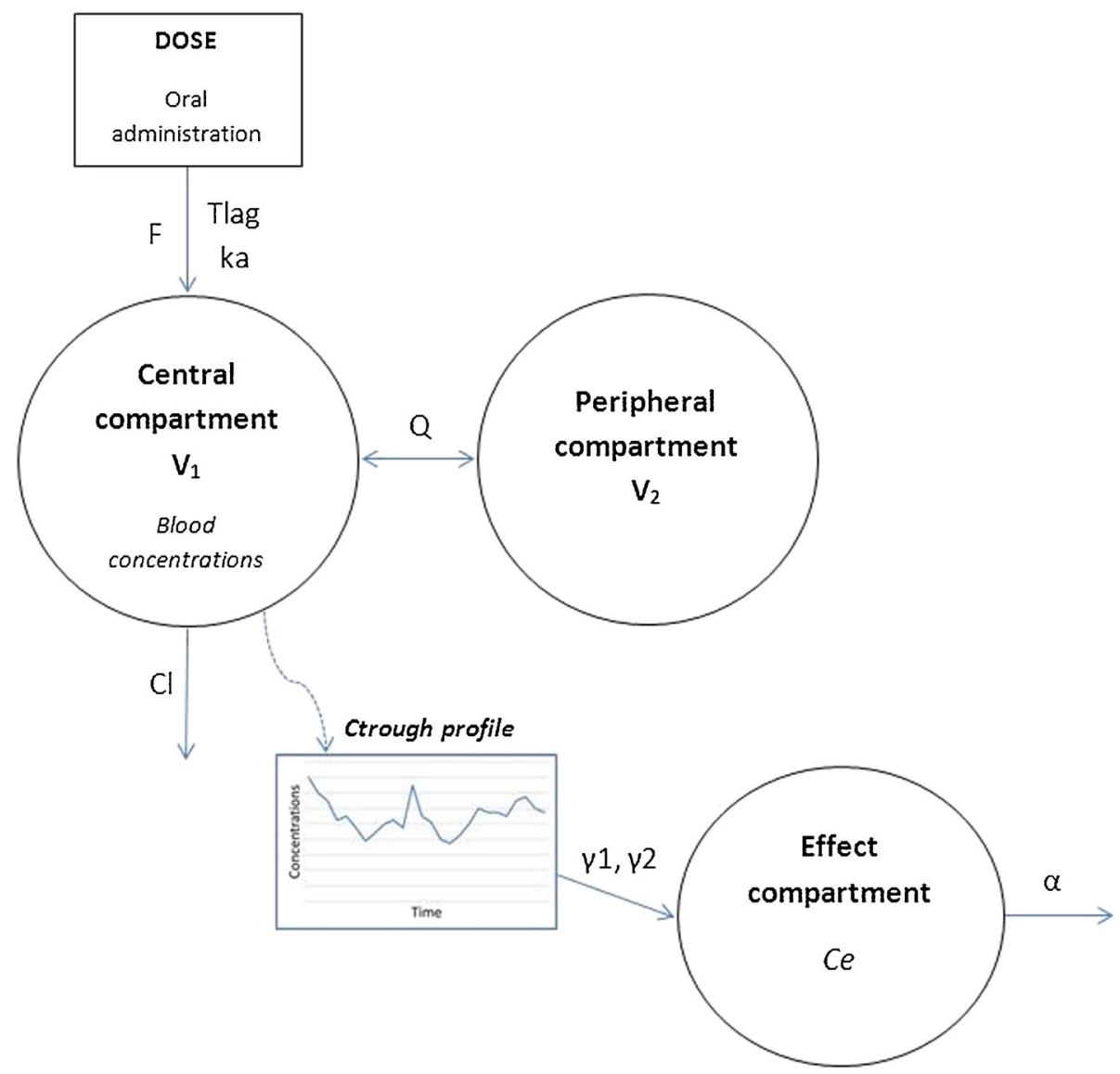

Fig. 1. Compartmental PK-PD model representation, with the Ctrough profile being the link between pharmacokinetics and pharmacodynamics

\section{Simulation-Based Determination of Target Concentrations}

With the therapeutic range being defined by the two thresholds $\gamma 1$ and $\gamma 2$, several scenarios were simulated to assess the impact of the CsA TBC distribution on TTR. CsA TBC distributions in populations of 100 patients were simulated, with different median values $(80,100,150$, and
$200 \mathrm{ng} / \mathrm{mL}$ ) and different coefficients of variation around the target $(10,20,30$, and 40\%). Fifty replications of 100-patient populations were simulated. The TTR distributions were compared between scenarios using Kaplan-Meier survival plots and descriptive statistics, to identify the optimal target of CsA TBC.

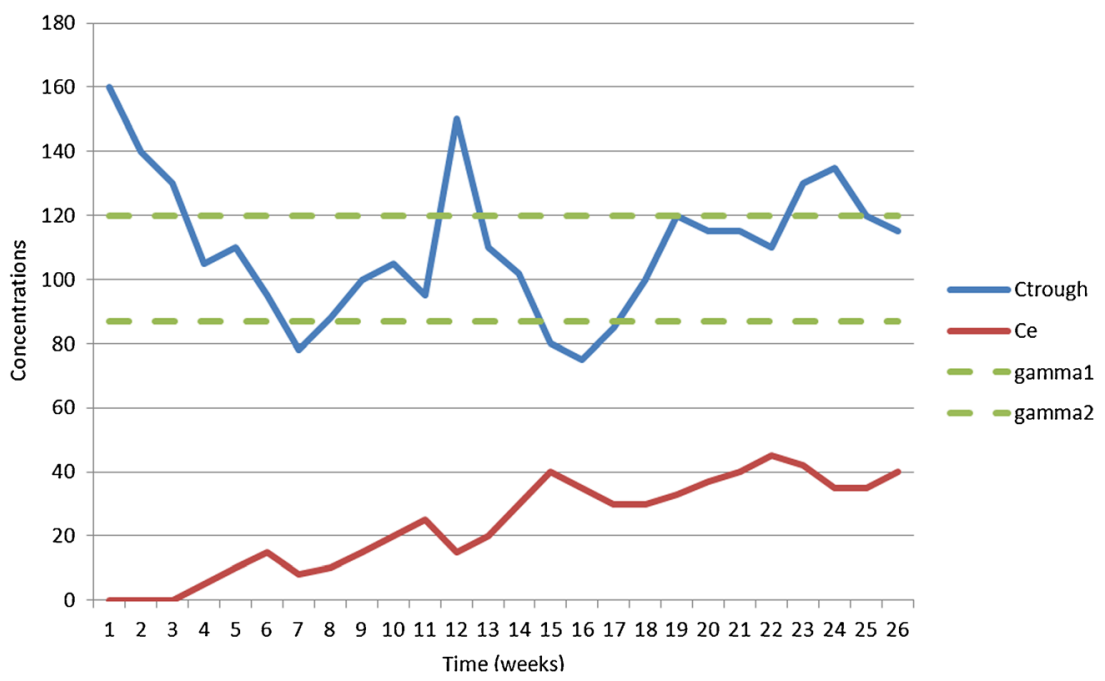

Fig. 2. Representation of the impact of TBC (Ctrough) and thresholds effective concentrations $(\gamma 1$ and $\gamma 2)$ on concentrations in the effect compartment $\left(C_{\mathrm{e}}\right)$ versus time 


\section{Application to New Patients}

Three patients newly diagnosed with SAA were prospectively considered for an external evaluation of the model. The history of CsA TBC was used as an input in the model to predict TTR (median and confidence interval). The predicted TTRs were compared to the observed responses.

\section{RESULTS}

\section{Patients}

Twenty-one patients hospitalized in the Pediatric Hematology and Oncology Units (20 in the Lyon center and one in the Grenoble center), corresponding to 23 courses, were included in the analysis. Patients' characteristics are summarized in Table I.

Fifteen patients had neutrophil response to IST with an average time of 69 days (range 19-182 days). Among them, nine responded on red blood cells (three patients had $\mathrm{Hb}$ $>80 \mathrm{~g} / \mathrm{L}$ at SAA diagnostic, three were transfusion dependent after a 6-month treatment) with a mean of 102 days (range 32-174 days), and seven responded on platelets with a mean of 79 days (range 39-149 days), whereas eight were still transfusion dependent after a 6-month treatment. One patient relapsed at day 515 and died from pulmonary post-graft complications.

Among the eight patients who had no neutrophil response, all were still hemoglobin and platelet transfusion dependent after a 6-month treatment. Five received a phenoidentical allograft from voluntary donors, and three patients received a second course of IST. One patient died at day 67 post-graft from respiratory distress syndrome and pulmonary hemorrhage.

Table I. Summary of Patients' Characteristics

\begin{tabular}{lll}
\hline & Mean & Min-max \\
\hline Number of patients-number of courses & $20-23$ & \\
Age (years) & 8.5 & $1-15$ \\
Sex (number of males/females) & $13 / 10$ & - \\
Weight (kg) & 34.0 & $9.8-79.3$ \\
BSA (m $\left.{ }^{2}\right)$ & 1.09 & $0.47-1.94$ \\
ANC (G/L) & 0.27 & $0-0.9$ \\
Platelets (G/L) & 29 & $2-94$ \\
Hemoglobin (g/L) & 92 & $55-128$ \\
Duration of follow-up (days) & 96 & $19-183$ \\
$\quad$ Neutrophil response to treatment & 69 & $19-182$ \\
$\quad$ No neutrophil response to treatment & 147 & $94-183$ \\
Number of CsA TBC per patient & 12 & $4-27$ \\
Response to treatment, number $(\%)$ & & - \\
$\quad$ Neutrophil-based response & $15(65.2 \%)$ & - \\
$\quad$ Hemoglobin-based response & $9(45.0 \%)$ & - \\
Platelet-based response & $7(34.4 \%)$ & - \\
Relapse & $3(13.0 \%)$ & \\
\hline
\end{tabular}

$B S A$ body surface area, $A N C$ absolute neutrophils count, $T B C$ trough blood concentrations

${ }^{a}$ Three patients were nonevaluable because of basal hemoglobin count $>80 \mathrm{~g} / \mathrm{L}$ at SAA diagnostic

\section{Pharmacokinetic Model}

Three hundred forty-one CsA TBC were available for the PK model development. The median number of measurements per patient in the dataset was 14 (range 7-27).

CsA TBC were adequately described by a twocompartment model with a linear elimination. Populationtypical PK parameters and associated IIV are listed in Table II.

All the parameters were well estimated, as shown by the relatively low values of relative standard errors. Residual variability was described by a combined proportional and additional error model. BW was found to influence the volume $\left(V_{1}, V_{2}\right)$ and clearance $(\mathrm{Cl}, Q)$ parameters, those parameters being allometrically scaled to weight as Eq. 4 with the allometric coefficient fixed to 0.75 for clearance parameters and 1 for volume parameters. No additional covariate was found as significantly improving the model.

The plot of model-based individual predictions of CsA TBC versus observations is shown in Fig. 3. Acceptable predictive performance was observed, with limited bias.

The profile of TBC (Ctrough) along the entire therapy was predicted for each individual and used as an input in the pharmacodynamic (PD) model. TBC was used as PK criterion because it is the monitoring criterion over time. This allows to define the effective range and the target concentration with respect to blood sampling in practice.

Table II. Cyclosporine Population Pharmacokinetic Parameters in SAA Children

Interindividual

Typical values (\%rse) variability $(\% \mathrm{CV})$

\begin{tabular}{|c|c|c|}
\hline \multicolumn{3}{|l|}{ PK parameter } \\
\hline$F(-)$ & 0.386 & 0 (fixed) \\
\hline Tlag (h) & 0.648 & 0 (fixed) \\
\hline $\mathrm{ka}\left(\mathrm{h}^{-1}\right)$ & 0.829 & 0 (fixed) \\
\hline$V_{1}\left(\mathrm{~L}(34 \mathrm{~kg})^{-1}\right)$ & $37.5(25)$ & 30 \\
\hline$Q\left(\mathrm{~L} \mathrm{~h}^{-1}(34 \mathrm{~kg})^{-0.75}\right)$ & $2.7(26)$ & 66.2 \\
\hline$V_{2}\left(\mathrm{~L}(34 \mathrm{~kg})^{-1}\right)$ & $1690(25)$ & 30 \\
\hline $\mathrm{Cl}\left(\mathrm{L} \mathrm{h}^{-1}(34 \mathrm{~kg})^{-0.75}\right)$ & $7.2(13)$ & 31.4 \\
\hline$a(\mathrm{mg} / \mathrm{L})$ & $0.03(9)$ & - \\
\hline$b(\%)$ & $25(9)$ & - \\
\hline \multicolumn{3}{|l|}{ TTR parameter } \\
\hline$\lambda_{0}\left(\mathrm{day}^{-1}\right)$ & $0.0027(58)$ & - \\
\hline$\alpha\left(\right.$ day $\left.^{-1}\right)$ & $0.028(107)$ & - \\
\hline Slope (mL/ng) & $17.2(102)$ & - \\
\hline$\gamma 1(\mathrm{ng} / \mathrm{mL})$ & $87(3)$ & - \\
\hline$\gamma 2(\mathrm{ng} / \mathrm{mL})$ & $120(3)$ & - \\
\hline
\end{tabular}

$F$ bioavailability, Tlag lag time, $k a$ absorption rate constant, $V_{1}$ volume of distribution of the central compartment, $V_{2}$ volume of distribution of the peripheral compartment, $Q$ intercompartment clearance, $\mathrm{Cl}$ total body clearance, $a$ additive error, $b$ proportional error, rse relative standard error, $C V$ coefficient of variation, $\lambda_{0}$ instantaneous hazard of response without treatment, $\alpha$ elimination rate constant of effective concentration, slope rate of increase of the instantaneous hazard for one unit increase of $C_{\mathrm{e}}, \gamma 1$ lower bound of CsA TBC, $\gamma 2$ upper bound of CsA TBC 


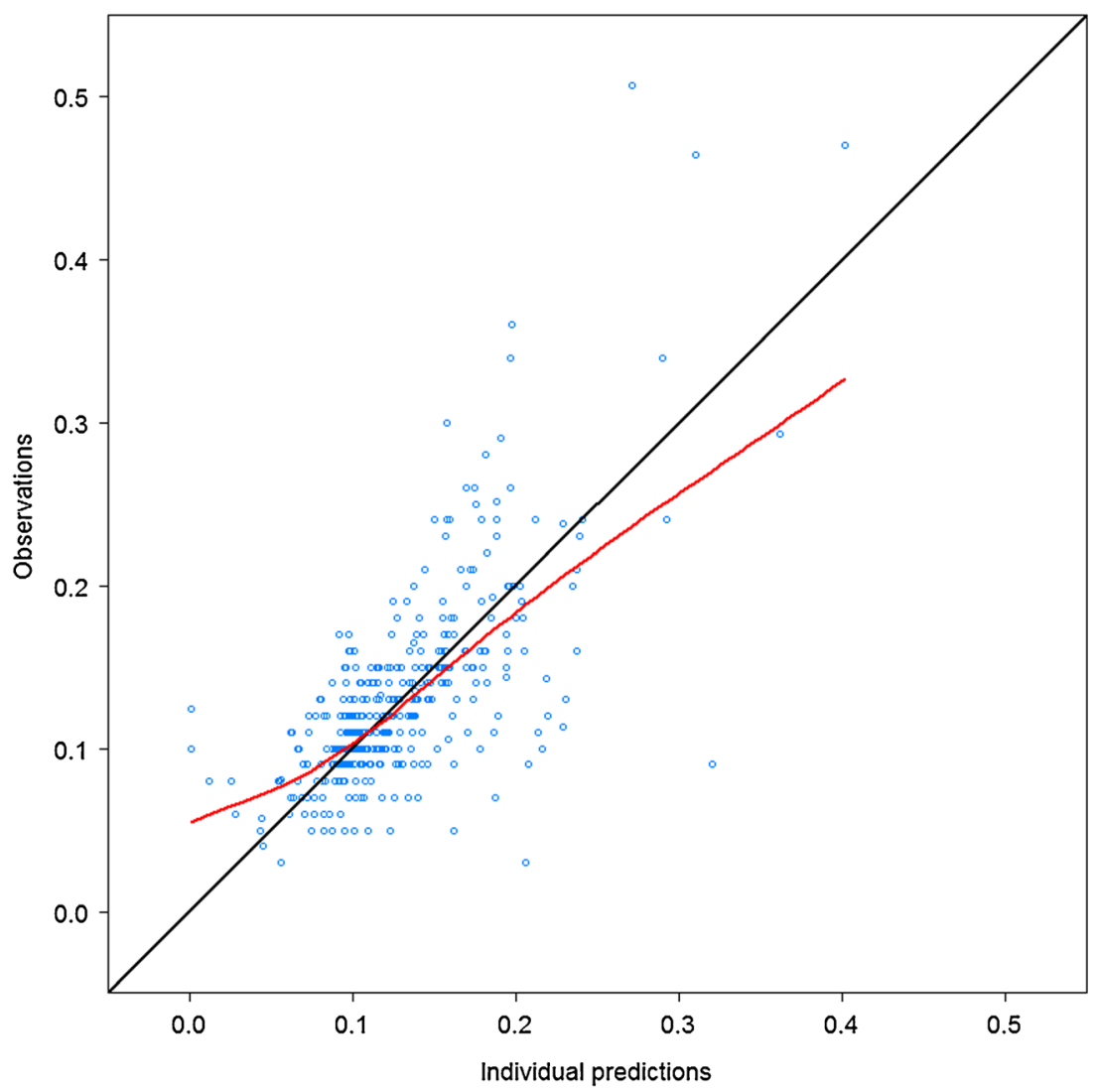

Fig. 3. Individual predictions versus observed concentrations. The black line is the identity line; the red line is the spline regression line (concentration unit is $\mu \mathrm{g} / \mathrm{mL}$ )

Time-to-Response Model and Definition of the Therapeutic Target Range

Estimated values for the TTR model parameters are shown in Table II. The estimated lower and upper bounds of the therapeutic target range ( $\gamma 1$ and $\gamma 2$ parameters) were 87 and $120 \mathrm{ng} / \mathrm{mL}$, respectively. Those values were estimated with very good precision (relative standard error $<5 \%$ ). The estimated half-life of the effective concentration (i.e., $\ln 2 / \alpha$ ) was 25 days. No IIV could be estimated on these parameters (IIV fixed to 0 for all the parameters).

Figure 4 represents the distribution of the model-based probability of response over time and the observed distribution of TTR. Given the limited number of individuals, the standard deviation of the probability density of the observed response is relatively large. The model satisfactorily described the probability of response to treatment in the patient group.

\section{Simulation-Based Determination of Target CsA TBC}

Simulations performed with the model using various target values showed that CsA TBC had a large impact on the probability of response to treatment (Fig. 5). The best probability of response was achieved with $100 \mathrm{ng} / \mathrm{mL}$ (Fig. 5b), which was in agreement with the $87-120-\mathrm{ng} / \mathrm{mL}$ range previously identified. Higher (150 or $200 \mathrm{ng} / \mathrm{mL}$ ) or lower $(80 \mathrm{ng} / \mathrm{mL}) \mathrm{CsA}$ TBC values were associated with a large decrease in the probability of response to treatment.
Simulations were performed considering several levels of variability (from 10 to $40 \%$, data not shown): when the target was $100 \mathrm{ng} / \mathrm{mL}$, increasing the variability would decrease the probability of response; when the target was either 80,150 , or $200 \mathrm{ng} / \mathrm{mL}$, increasing the variability would improve this probability since more concentrations have the chance to fall within the therapeutic interval.

\section{Application to New Patients}

TTRs were predicted for three additional patients, based on the proposed model and the observed CsA TBC. Patients' characteristics are summarized in Table III. For the three patients, TTR-predicted distribution was remarkably consistent with the observations (Fig. 6). Although these validation data are very limited, the model performance in predicting TTR was satisfactory.

\section{DISCUSSION}

SAA is a rare and potentially fatal disease that results in pancytopenia and hypocellular bone marrow $(28,29)$. This work has broad scientific importance in SAA treatment. The first-line treatment is genoidentical BMT. However, in two thirds of cases, no suitable donor is available and IST becomes necessary. The response rate to IST varies from 60 to $80 \%$ with a survival rate at 5 years ranging from 55 to $80 \%$ $(7,9)$. Hematological response rarely occurs before 4 months. 


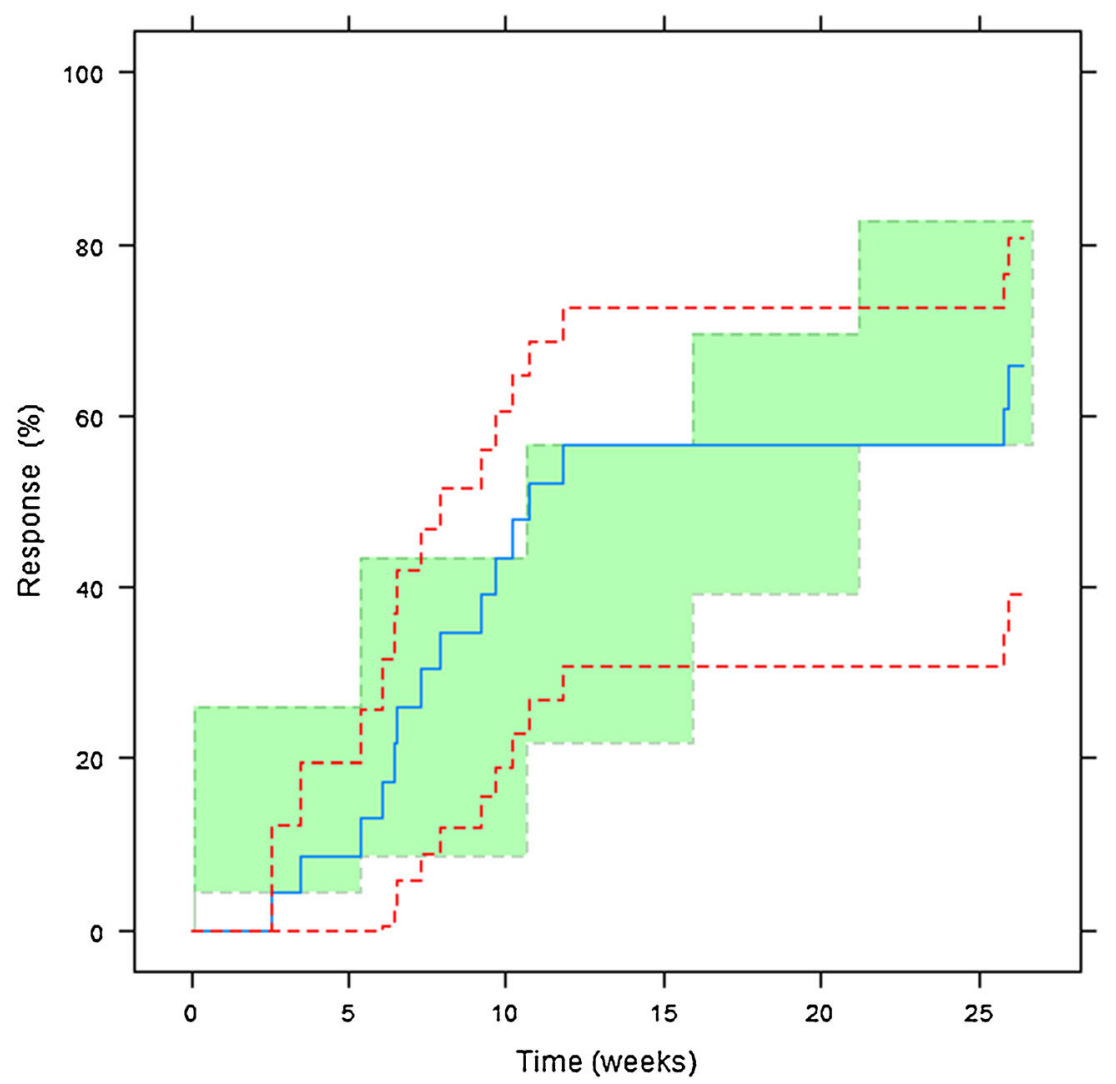

Fig. 4. Visual predictive checks of the probability of response over time. The median (solid blue line) and the 5th and 95th percentiles (dashed red lines) of the observed data are in accordance with the $90 \%$ confidence interval of the TTR estimated by the model (green area) based on 100 replicates of the 23 original patients

Disease-free survival is estimated at only $40 \%$ at 10 years (30). Apart from unrelated BMT, few alternatives are available for nonresponders to IST. But transplantationrelated mortality after unrelated BMT is not negligible, with a 5-year overall survival of $51 \%$ (31). Increasing the probability of response to IST might permit to avoid numerous and hazardous BMT, with a positive impact on supporting care costs.

Because of its large PK variability and its toxicity profile, CsA presents a narrow therapeutic index. Current guidelines recommend a target $\mathrm{TBC}$ range somewhat large, as high as 200 to $400 \mathrm{ng} / \mathrm{mL}$ (8). The choice of these values has not been supported by any PK/PD data.

In recent years, new data on SAA physiopathology have been published. It has been shown that a T-lymphocyte subpopulation, Treg, is involved as in many other autoimmune diseases. Solomou et al. (12) have shown that the number of Treg was decreased in patients with aplastic anemia. More recently, Sutton et al. (16) demonstrated that Treg were a good prognostic factor for response and severity of aplastic anemia. In addition, it has been shown that the effect of CsA on Treg depends on its concentration. Low CsA concentrations allow Treg expansion, whereas high concentrations lead to a decreased Treg number $(17,32,33)$. This phenomenon can be explained through interleukin-2 (IL-2) action. CD4+ T-cell differentiation in Treg involves moderate concentrations of IL-2. However, IL-2 production is inversely related to CsA concentrations (34). Insufficient exposure to
IL-2 caused by high CsA concentration may result in the inhibition of Treg formation. But excessive exposure to IL-2 due to very low CsA concentrations may compromise Treg expansion. This assumption is also supported by some studies establishing that only ultralow doses of IL-2 are necessary to a better expansion of Treg (35-37).

The involvement of Treg and the concentrationdependent effect of CsA on IL-2 inhibition suggest that there may exist an effective range of CsA exposure in the treatment of SAA, but it has not been identified so far in clinical practice. The main result of this study is consistent with the relationships between Treg, IL-2, and CsA described above. We used mathematical modeling to investigate the relationships between CsA exposure and treatment response based on neutrophil count in 20 children (during 23 treatment courses) with SAA. Then, we used this model to determine the range of CsA TBC associated with optimal response.

To date, no relationship has been established between the PK and the PD of CsA in SAA, and no PK model of CsA has been presented in this disease so far. This is also the first study using a quantitative, model-based approach of CsA optimization in children with SAA.

The PK model was a two-compartment model. This is consistent with previous PK studies of CsA in children $(24,38)$. The estimated parameter values were comparable to those found in the literature $(24,39)$.

The proposed model has been linked to TTR after CsA treatment initiation. This approach is quite original, as few 

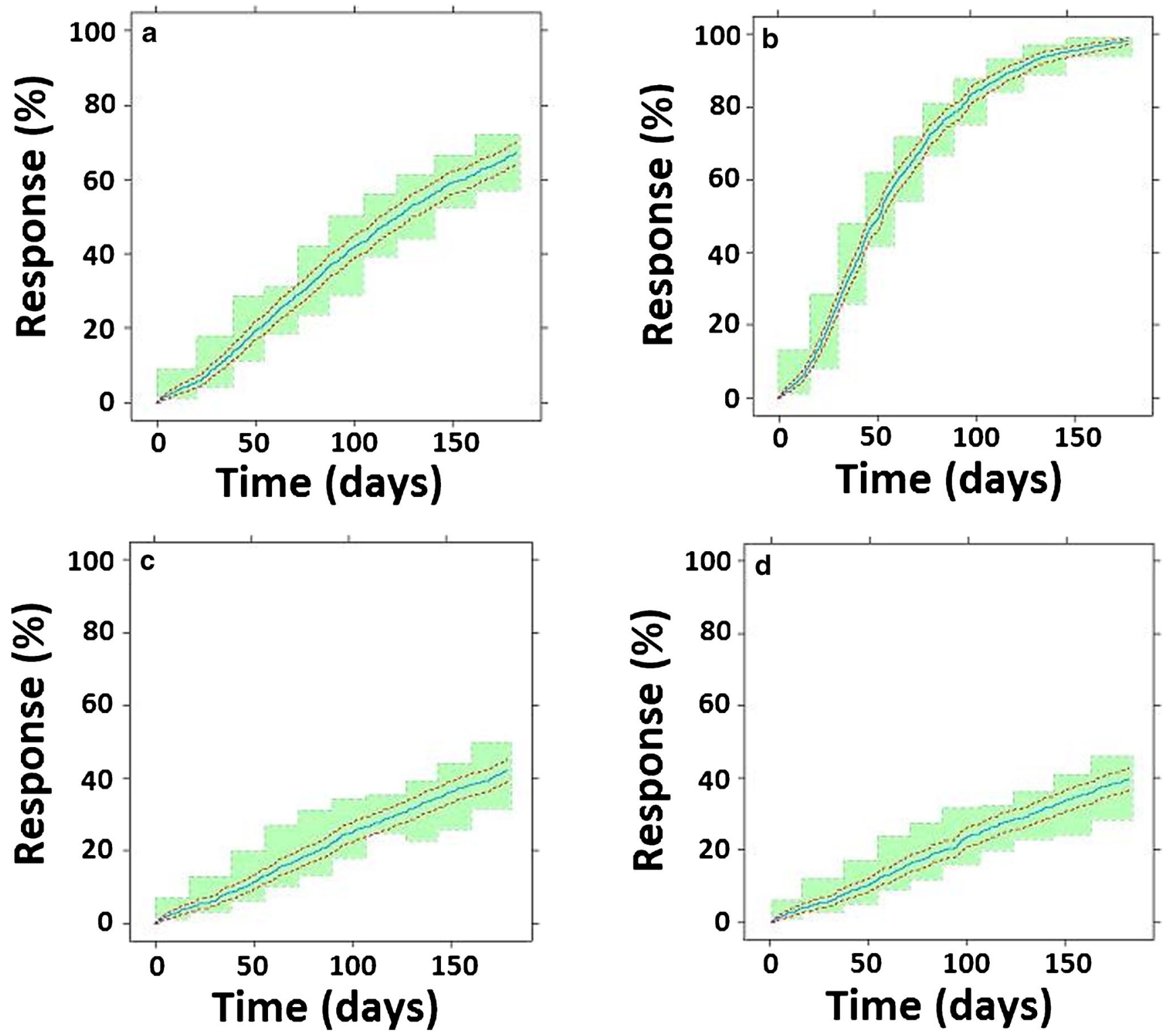

Fig. 5. Profiles of simulated probability of treatment response with different TBC targets: 80 (a), 100 (b), 150 (c), and $200 \mathrm{ng} / \mathrm{mL}(\mathbf{d})$ with a variability of $10 \%$. The median (solid blue line) and the 5th and 95th percentiles (dashed red lines) of the observed data are in accordance with the $90 \%$ confidence interval of the TTR estimated by the model (green area)

complex models were used to assess TTR under treatment in rare diseases. Other models, such as static logistic model to compare time-to-response according to the average TBC, have been used but did not allow taking into account the dynamics of TBC values with time within each patient nor defining an efficient range of concentration.

This work was based on data collected during routine patients' care. In this setting, only trough concentration was available, which is a rude surrogate of CsA exposure, but rich sampling was impossible in these children. However, the PKPD model fulfilled the objective of describing CsA TBC and the time course of the response in the study population and predicting the response in three additional patients. TTR was linked to CsA TBC via an interface model. Similar to the so- called effect compartment (40), the interface model is a virtual compartment, directly linked to the effect but mimicking a delay in the effect. This is exemplified by the difference between the estimated half-life of the effective concentration (about 25 days) and CsA half-life in blood (about $2.6 \mathrm{~h}$ ). Moreover, the interface approach allowed us to state and to estimate a therapeutic window for CsA exposure. We estimated that a $87-120-\mathrm{ng} / \mathrm{mL}$ range of CsA TBC was optimal for response to treatment in this patient population. The boundaries of this range were estimated with very good precision. Our results suggest that current recommendations regarding the use of CsA in SAA should be revised and that lower doses and CsA exposure should be investigated in clinical studies. Although our findings need to be confirmed 
Table III. Summary of Patients' Characteristics for External Evaluation

\begin{tabular}{|c|c|c|c|}
\hline & Patient no. 1 & Patient no. 2 & Patient no. 3 \\
\hline Age (years) & 4 & 15 & 5 \\
\hline Sex & $\mathrm{F}$ & $\mathrm{F}$ & M \\
\hline Weight $(\mathrm{kg})$ & 15.1 & 46 & 29.8 \\
\hline \multicolumn{4}{|l|}{ Blood cell counts at diagnostic } \\
\hline ANC $(\mathrm{G} / \mathrm{L})$ & 0.3 & 0.3 & 0.2 \\
\hline Platelets $(\mathrm{G} / \mathrm{L})$ & 2 & 2 & 8 \\
\hline Hemoglobin $(\mathrm{g} / \mathrm{L})$ & 40 & 98 & 85 \\
\hline Duration of follow-up (days) & 457 & 425 & 105 \\
\hline Target CsA TBC (ng/mL) & 100 & 100 & 100 \\
\hline Number of CsA TBC per patient & 20 & 15 & 13 \\
\hline Mean CsA $\mathrm{TBC} \pm \mathrm{SD}(\mathrm{ng} / \mathrm{mL})$ until ANC response & $99.7 \pm 23.8$ & $104.4 \pm 40.1$ & $112.0 \pm 28.1$ \\
\hline \multicolumn{4}{|l|}{ Treatment response (days) } \\
\hline $\mathrm{ANC}$ & 57 & 41 & 61 \\
\hline Platelets $^{\mathrm{a}}$ & 280 & 265 & 93 \\
\hline Hemoglobin ${ }^{\mathrm{a}}$ & 300 & 80 & $\mathrm{No}^{\mathrm{b}}$ \\
\hline Relapse & No & No & No \\
\hline
\end{tabular}

$A N C$ absolute neutrophils count, $T B C$ trough blood concentrations, $S D$ standard deviation

${ }^{a}$ Treatment response defined as transfusion independence and platelets $>20 \mathrm{G} / \mathrm{L}$ or hemoglobin $>80 \mathrm{~g} / \mathrm{L}$

${ }^{b}$ Transfusion dependence at day 105

in a larger patient population, they are in agreement with the biological rationale of CsA action on Treg explained above and our experience with salvage therapy of refractory SAA (18). By decreasing CsA exposure, fewer toxic events (e.g., nephrotoxicity, hypertension) could be expected. However, our study was not designed to investigate cyclosporine tolerance. Further randomized studies are needed to evaluate the potential benefit of low CsA TBC on toxicity.

Although the model performance was satisfactory, there are several limitations in this work. Model building and validation were limited by a small number of patients. The rarity of this disease justifies the apparently small number of patients included in this analysis. Moreover, this was a retrospective study. Our results need to be confirmed in a large prospective cohort of children with SAA. Finally, we used ANC as response criterion, while usual criteria include hemoglobin, platelets, and ANC (19). Several reasons can justify this choice: a neutrophil response allows children discharge from hospital. While red blood cells and platelets can be supplemented, growth factors have no impact on neutrophil response (41). Also, neutrophils usually respond first, then red blood cell and platelets. In our study, average time of response was 69 days for ANC, 79 for platelets, and 102 for red blood cells. Finally, from a modeling point of view, including criteria based on erythrocytes and platelet values would have confounded any PK-PD analysis because of numerous transfusions (autologous erythrocytes could not be identified).

\section{CONCLUSION}

Using PK-PD modeling, we described the relationships between CsA TBC and the time course of neutrophil response to treatment in children with SAA. An efficient target range of CsA TBC was derived from the model. This range $(87-120 \mathrm{ng} / \mathrm{mL})$ suggests that CsA concentrations lower than those currently recommended may be associated with a better rate of response
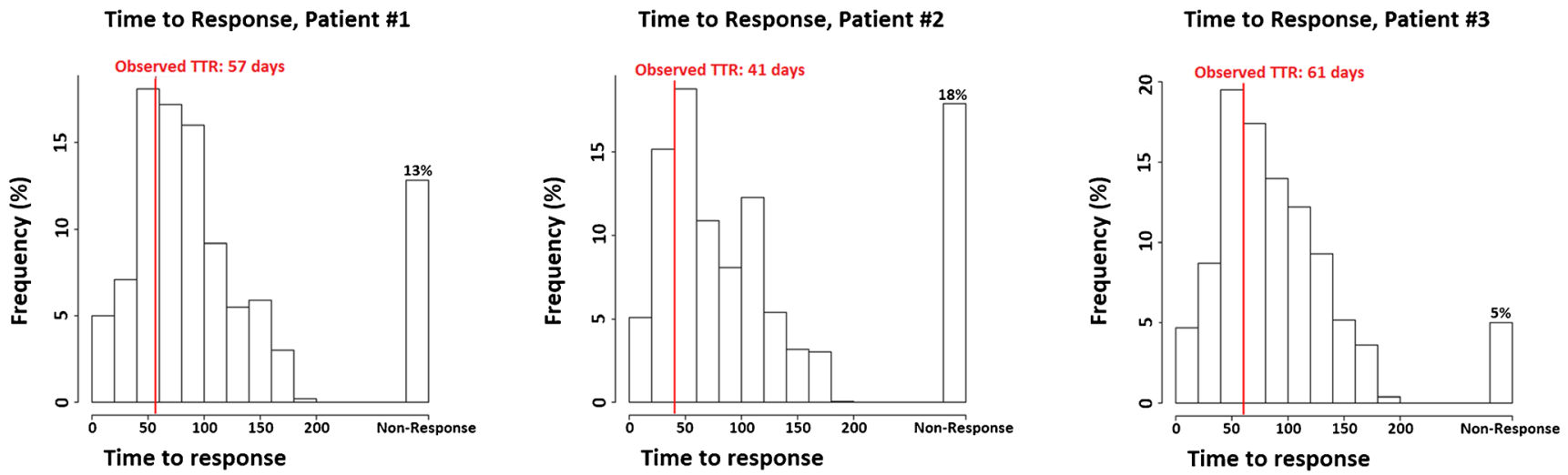

Fig. 6. Predicted distribution of the TTR (histogram) and observed TTR (red line) in three external patients (i.e., not included for the model development) 
on ANC in children with SAA. Those findings need to be confirmed in a larger, prospective clinical study.

\section{ACKNOWLEDGMENTS}

The authors would like to thank Professor Michel Tod, Hospices Civils de Lyon, Faculty of Pharmacy of Lyon, and University Lyon 1 for helpful advices.

\section{Compliance with Ethical Standards}

Conflict of Interest The authors declare that they have no conflict of interest.

For this type of study, formal consent is not required.

\section{REFERENCES}

1. Issaragrisil S, Kaufman DW, Anderson T, Chansung K, Leaverton PE, Shapiro S, et al. The epidemiology of aplastic anemia in Thailand. Blood. 2006;107(4):1299-307.

2. Montané E, Ibáñez L, Vidal X, Ballarín E, Puig R, García N, et al. Epidemiology of aplastic anemia: a prospective multicenter study. Haematologica. 2008;93(4):518-23.

3. Marsh JCW, Ball SE, Cavenagh J, Darbyshire P, Dokal I, GordonSmith EC, et al. Guidelines for the diagnosis and management of aplastic anaemia. Br J Haematol. 2009;147(1):43-70.

4. Frickhofen N, Heimpel H, Kaltwasser JP, Schrezenmeier H. Antithymocyte globulin with or without cyclosporin A: 11-year follow-up of a randomized trial comparing treatments of aplastic anemia. Blood. 2003;101(4):1236-42.

5. Bacigalupo A, Bruno B, Saracco P, Bona ED, Locasciulli A, Locatelli $\mathrm{F}$, et al. Antilymphocyte globulin, cyclosporine, prednisolone, and granulocyte colony-stimulating factor for severe aplastic anemia: an update of the GITMO/EBMT study on 100 patients. Blood. 2000;95(6):1931-4.

6. Rosenfeld S, Follmann D, Nunez O, Young NS. Antithymocyte globulin and cyclosporine for severe aplastic anemia: association between hematologic response and long-term outcome. JAMA J Am Med Assoc. 2003;289(9):1130-5.

7. Kojima S, Horibe K, Inaba J, Yoshimi A, Takahashi Y, Kudo K, et al. Long-term outcome of acquired aplastic anaemia in children: comparison between immunosuppressive therapy and bone marrow transplantation. Br J Haematol. 2000;111(1):321-8.

8. Scheinberg P, Nunez O, Weinstein B, Scheinberg P, Biancotto A $\mathrm{Wu} \mathrm{CO}$, et al. Horse versus rabbit antithymocyte globulin in acquired aplastic anemia. N Engl J Med. 2011;365(5):430-8.

9. Führer M, Rampf U, Baumann I, Faldum A, Niemeyer C, JankaSchaub G, et al. Immunosuppressive therapy for aplastic anemia in children: a more severe disease predicts better survival. Blood. 2005;106(6):2102-4.

10. Frickhofen N, Rosenfeld SJ. Immunosuppressive treatment of aplastic anemia with antithymocyte globulin and cyclosporine. Semin Hematol. 2000;37(1):56-68.

11. Young NS. Autoimmunity and its treatment in aplastic anemia. Ann Intern Med. 1997;126(2):166-8.

12. Solomou EE, Rezvani K, Mielke S, Malide D, Keyvanfar K, Visconte $\mathrm{V}$, et al. Deficient CD4+ CD25+ FOXP3+ T regulatory cells in acquired aplastic anemia. Blood. 2007;110(5):1603-6.

13. Wang X-G, Wang M, Liu S, Wang X-G, Qiao J-Y, Cao Y-M, et al. Effect of cyclosporine on regulatory $\mathrm{T}$ cells and Foxp3 in the peripheral blood of children with chronic aplastic anemia. Zhongguo Dang Dai Er Ke Za Zhi Chin J Contemp Pediatr. 2011;13(12):936-9.

14. Wang X-G, Cao Y-M, Wang X-G. Detection and significance of $\mathrm{CD} 4+\mathrm{CD} 25$ int/high $\mathrm{CD} 127$ low regulatory $\mathrm{T}$ cells in pediatric aplastic anemia. Zhongguo Dang Dai Er Ke Za Zhi Chin J Contemp Pediatr. 2011;13(4):292-5.

15. Kordasti S, Marsh J, Al-Khan S, Jiang J, Smith A, Mohamedali $\mathrm{A}$, et al. Functional characterization of CD4+ T cells in aplastic anemia. Blood. 2012;119(9):2033-43.

16. Sutton KS, Shereck EB, Nemecek ER, Kurre P. Immune markers of disease severity and treatment response in pediatric acquired aplastic anemia. Pediatr Blood Cancer. 2013;60(3):455-60.

17. Kawai M, Kitade H, Mathieu C, Waer M, Pirenne J. Inhibitory and stimulatory effects of cyclosporine A on the development of regulatory T cells in vivo. Transplantation. 2005;79(9):1073-7.

18. Bertrand A, Philippe M, Bertrand Y, Plantaz D, Bleyzac N. Salvage therapy of refractory severe aplastic anemia by decreasing cyclosporine dose regimen. Eur J Haematol. 2014;92(2):172-6.

19. Camitta BM, Thomas ED, Nathan DG, Gale RP, Kopecky KJ, Rappeport JM, et al. A prospective study of androgens and bone marrow transplantation for treatment of severe aplastic anemia. Blood. 1979;53(3):504-14.

20. French Public Health Code - Article R1121-2. Code de la santé publique.

21. Lavielle M, Mentré F. Estimation et planification dans les modèles non linéaires à effets mixtes. Application à la dynamique du VIH sous traitement. 2009 [cité 10 avr 2014]. Disponible sur: http://hal.inria.fr/inria-00386791.

22. Kuhn E, Lavielle M. Maximum likelihood estimation in nonlinear mixed effects models. Comput Stat Data Anal. 2005;49(4):1020-38.

23. Lavielle M, Mentré F. Estimation of population pharmacokinetic parameters of saquinavir in HIV patients with the MONOLIX software. J Pharmacokinet Pharmacodyn. 2007;34(2):229-49.

24. Willemze AJ, Cremers SC, Schoemaker RC, Lankester AC, Den Hartigh J, Burggraaf $\mathrm{J}$, et al. Ciclosporin kinetics in children after stem cell transplantation. Br J Clin Pharmacol. 2008;66(4):539-45.

25. Meille C, Iliadis A, Barbolosi D, Frances N, Freyer G. An interface model for dosage adjustment connects hematotoxicity to pharmacokinetics. J Pharmacokinet Pharmacodyn. 2008;35(6):619-33.

26. Holford N. A time to event tutorial for pharmacometricians. CPT Pharmacomet Syst Pharmacol. 2013;2(5), e43.

27. Yano Y, Beal SL, Sheiner LB. Evaluating pharmacokinetic/ pharmacodynamic models using the posterior predictive check. J Pharmacokinet Pharmacodyn. 2001;28(2):171-92.

28. Brodsky RA, Jones RJ. Aplastic anaemia. Lancet. 2005;365(9471):1647-56.

29. Young NS, Calado RT, Scheinberg P. Current concepts in the pathophysiology and treatment of aplastic anemia. Blood. 2006;108(8):2509-19.

30. Kojima S, Hibi S, Kosaka Y, Yamamoto M, Tsuchida M, Mugishima $\mathrm{H}$, et al. Immunosuppressive therapy using antithymocyte globulin, cyclosporine, and danazol with or without human granulocyte colony-stimulating factor in children with acquired aplastic anemia. Blood. 2000;96(6):2049-54.

31. Perez-Albuerne ED, Eapen M, Klein J, Gross TJ, Lipton JM, Baker KS, et al. Outcome of unrelated donor stem cell transplantation for children with severe aplastic anemia. $\mathrm{Br} \mathrm{J}$ Haematol. 2008;141(2):216-23.

32. Brandt C, Pavlovic V, Radbruch A, Worm M, Baumgrass R. Low-dose cyclosporine A therapy increases the regulatory $\mathrm{T}$ cell population in patients with atopic dermatitis. Allergy. 2009;64(11):1588-96.

33. López-Flores R, Bojalil R, Benítez JC, Ledesma-Soto Y, Terrazas $\mathrm{CA}$, Rodríguez-Sosa $\mathrm{M}$, et al. Consecutive low doses of cyclosporine A induce pro-inflammatory cytokines and accelerate allograft skin rejection. Mol Basel Switz. 2011;16(5):3969-84.

34. Stein CM, Murray JJ, Wood AJ. Inhibition of stimulated interleukin-2 production in whole blood: a practical measure of cyclosporine effect. Clin Chem. 1999;45(9):1477-84.

35. Kennedy-Nasser AA, Ku S, Castillo-Caro P, Hazrat Y, Wu M-F, Liu H, et al. Ultra low-dose IL-2 for GVHD prophylaxis after allogeneic hematopoietic stem cell transplantation mediates expansion of regulatory $\mathrm{T}$ cells without diminishing antiviral and antileukemic activity. Clin Cancer Res Off J Am Assoc Cancer Res. 2014;20(8):2215-25. 
36. Saadoun D, Rosenzwajg M, Joly F, Six A, Carrat F, Thibault V, et al. Regulatory T-cell responses to low-dose interleukin-2 in HCV-induced vasculitis. N Engl J Med. 2011;365(22):2067-77.

37. Ito S, Bollard CM, Carlsten M, Melenhorst JJ, Biancotto A, Wang E, et al. Ultra-low dose interleukin-2 promotes immune-modulating function of regulatory $\mathrm{T}$ cells and natural killer cells in healthy volunteers. Mol Ther J Am Soc Gene Ther. 2014;22(7):1388-95.

38. Bourgoin H, Paintaud G, Buchler M, Lebranchu Y, Autret-Leca E, Mentre F, et al. Bayesian estimation of cyclosporin exposure for routine therapeutic drug monitoring in kidney transplant patients. Br J Clin Pharmacol. 2005;59(1):18-27.

39. Fanta S, Jönsson S, Backman JT, Karlsson MO, Hoppu K. Developmental pharmacokinetics of ciclosporin-a population pharmacokinetic study in paediatric renal transplant candidates. Br J Clin Pharmacol. 2007;64(6):772-84.

40. Sheiner LB, Stanski DR, Vozeh S, Miller RD, Ham J. Simultaneous modeling of pharmacokinetics and pharmacodynamics: application to d-tubocurarine. Clin Pharmacol Ther. mars 1979;25(3):358-71.

41. Tichelli A, Schrezenmeier H, Socié G, Marsh J, Bacigalupo A, Dührsen U, et al. A randomized controlled study in patients with newly diagnosed severe aplastic anemia receiving antithymocyte globulin (ATG), cyclosporine, with or without G-CSF: a study of the SAA Working Party of the European Group for Blood and Marrow Transplantation. Blood. 2011;117(17):443441. 